\title{
Uniqueness of positive solutions for the singular fractional differential equations involving integral boundary value conditions
}

\section{Dandan Min' ${ }^{1}$ Lishan $\mathrm{Liu}^{1,2^{*}}$ and Yonghong $\mathrm{Wu}^{2}$}

${ }^{*}$ Correspondence:

mathlls@163.com

${ }^{1}$ School of Mathematical Sciences,

Qufu Normal University, Qufu ,

People's Republic of China

${ }^{2}$ Department of Mathematics and

Statistics, Curtin University, Perth,

Australia

\begin{abstract}
This paper is concerned with the uniqueness of positive solutions for a class of singular fractional differential equations with integral boundary conditions. The nonlinear term and boundary conditions of fractional differential equation contain the fractional order derivatives. The uniqueness of positive solutions is derived by the fixed point theorem of mixed monotone operator. An example is given to demonstrate the validity of our main results.
\end{abstract}

MSC: 26A33; 34A08; 34B15; 34B16; 34B18

Keywords: Fractional differential equations; Integral boundary value problem; Uniqueness of positive solutions; Singularity; Mixed monotone operator

\section{Introduction}

In this paper, we investigate the uniqueness of positive solutions for the following class of nonlinear singular fractional differential equations:

$$
\left\{\begin{array}{l}
D_{0^{+}}^{\alpha} u(t)+f\left(t, u(t), D_{0^{+}}^{\alpha_{1}} u(t), D_{0^{+}}^{\alpha_{2}} u(t), \ldots, D_{0^{+}}^{\alpha_{n-2}} u(t)\right)=0, \quad t \in(0,1), \\
u(0)=D_{0^{+}}^{\gamma_{1}} u(0)=D_{0^{+}}^{\gamma_{2}} u(0)=\cdots=D_{0^{+}}^{\gamma_{n-2}} u(0)=0, \\
D_{0^{+}}^{\beta_{1}} u(1)=\int_{0}^{\eta} h(s) D_{0^{+}}^{\beta_{2}} u(s) d A(s)+\int_{0}^{1} a(s) D_{0^{+}}^{\beta_{3}} u(s) d A(s),
\end{array}\right.
$$

where $D_{0^{+}}^{\alpha} u, D_{0^{+}}^{\alpha_{k}} u, D_{0^{+}}^{\gamma_{k}} u(k=1,2, \ldots, n-2)$, and $D_{0^{+}}^{\beta_{i}} u(i=1,2,3)$ are the standard RiemannLiouville derivatives and $n-1<\alpha \leq n, k-1<\alpha_{k}, \gamma_{k} \leq k(k=1,2, \ldots, n-2), n-k-1<$ $\alpha-\alpha_{k} \leq n-k, n-k-1<\alpha-\gamma_{k} \leq n-k(k=1,2, \ldots, n-2), \gamma_{n-2}-\alpha_{n-2} \geq 0, \beta_{1} \geq \beta_{2}, \beta_{1} \geq \beta_{3}$, $\alpha-\beta_{i}>1, \beta_{i}-\alpha_{n-2} \geq 0(i=1,2,3), f:(0,1) \times(0,+\infty)^{n-1} \rightarrow \mathbb{R}_{+}^{1}=[0,+\infty)$ is continuous and $a, h \in C(0,1), A$ is a function of bounded variation, $\int_{0}^{\eta} h(s) D_{0^{+}}^{\beta_{2}} u(s) d A(s)$, $\int_{0}^{1} a(s) D_{0^{+}}^{\beta_{3}} u(s) d A(s)$ denote the Riemann-Stieltjes integral with respect to $A$.

Fractional differential equations arise in the mathematical modeling of systems and processes occurring in many engineering and scientific disciplines such as physics, chemistry, polymer rheology, control theory, diffusive transport akin to diffusion, electrical networks, probability, etc. For details, see [1-5] and the references therein. Fractional-order differential equations and integral operators are found to be a better tool for the description of

(c) The Author(s) 2018. This article is distributed under the terms of the Creative Commons Attribution 4.0 International License (http://creativecommons.org/licenses/by/4.0/), which permits unrestricted use, distribution, and reproduction in any medium, provided you give appropriate credit to the original author(s) and the source, provide a link to the Creative Commons license, and indicate if changes were made. 
hereditary properties of various materials and processes than the corresponding integerorder differential equations. The topic of fractional-order boundary value problems has been intensively studied by several researchers, see [1-31] and the references therein, and a great deal of results have been developed for differential and integral boundary value problems. The existence and uniqueness of positive solutions for such problems have become an important area of investigation in recent years. These studies mainly use the fixed point theory in cones.

In [6], Bai and Sun investigated the following fractional differential equations:

$$
\left\{\begin{array}{l}
D_{0^{+}}^{\alpha} u(t)+f\left(t, u(t), D_{0^{+}}^{v} u(t)\right)=0, \quad t \in(0,1) \\
u(0)=u^{\prime}(0)=u^{\prime \prime}(0)=u^{\prime \prime}(1)=0
\end{array}\right.
$$

where $\alpha, v, \mu \in \mathbb{R}_{+}^{1}, 3<\alpha \leq 4,0<v \leq 1$ are real numbers, $f:[0,1] \times \mathbb{R}^{3} \rightarrow \mathbb{R}^{1}=$ $(-\infty,+\infty)$ satisfies the local Carathéodory conditions, $f(t, x, y, z)$ is singular at $x, y, z=0$, and $D_{0^{+}}^{v}, D_{0^{+}}^{\mu}, D_{0^{+}}^{\alpha}$ are the Riemann-Liouville fractional derivatives. The authors obtained the existence and multiplicity of positive solutions by means of Krasnosel'skii's fixed point.

In [7], by means of a fixed point theorem of the mixed monotone operator, Zhang et al. investigated the uniqueness of positive solutions for the fractional differential equation with integral boundary conditions:

$$
\begin{cases}-D_{t}^{\beta}\left(\varphi_{p}\left(-D_{t}^{\alpha} x\right)\right)(t)=f\left(x(t), D_{t}^{\gamma} x(t)\right), & t \in(0,1), \\ D_{t}^{\alpha} x(0)=D_{t}^{\alpha+1} x(0)=D_{t}^{\alpha} x(1)=0, & D_{t}^{\gamma} x(0)=0, \quad D_{t}^{\gamma} x(1)=\int_{0}^{1} D_{t}^{\gamma} x(s) d A(s),\end{cases}
$$

where $\alpha, v, \mu \in \mathbb{R}_{+}^{1}, 0<\gamma \leq 1<\alpha \leq 2<\beta<3, \alpha-\gamma>1, D_{t}^{\alpha}, D_{t}^{\beta}, D_{t}^{\gamma}$ are the standard Riemann-Liouville derivatives, $\int_{0}^{1} x(s) d A(s)$ is denoted by a Riemann-Stieltjes integral, $A$ is a function of bounded variation, and $d A$ can be a signed measure. The $p$-Laplacian operator is defined as $\varphi_{p}(s)=|s|^{p-2} s, p>2$, and $f(t, u, v)$ may be singular at both $u=0$ and $v=0$.

In [9], Cabada and Wang studied the existence of positive solutions of the following nonlinear factional differential equation with integral boundary conditions:

$$
\left\{\begin{array}{l}
D_{0^{+}}^{\alpha} u(t)+f(t, u(t))=0, \quad 0<t<1 \\
u(0)=u^{\prime}(0)=0, \quad u(1)=\lambda \int_{0}^{1} u(s) d s
\end{array}\right.
$$

where $2<\alpha \leq 3,0<\lambda, \lambda \neq \alpha, D_{0^{+}}^{\alpha}$ is the standard Riemann-Liouville fractional derivative, and $f:[0,1] \times \mathbb{R}_{+}^{1} \rightarrow \mathbb{R}_{+}^{1}$ is a continuous function.

In [12], by means of fixed point theory, Qarout et al. investigated the existence and uniqueness of solutions for the following nonlinear fractional differential equation with multi-point strip boundary conditions:

$$
\left\{\begin{array}{l}
{ }^{c} D_{0^{+}}^{q} x(t)=f(t, x(t)), \quad n-1<q \leq n, 0 \leq t \leq 1, \\
x(0)=x^{\prime}(0)=x^{\prime \prime}(0)=\cdots=x^{n-2}(0)=0, \\
x(1)=a \int_{0}^{\xi} x(s) d s+b \sum_{i=1}^{m-2} \alpha_{i} x\left(\eta_{i}\right),
\end{array}\right.
$$


where ${ }^{c} D_{0^{+}}^{\alpha}$ denote the Caputo fractional derivative of order $q, 0<\xi<\eta_{1}<\eta_{2}<\cdots<\eta_{n-2}<$ $1, f:[0,1] \times \mathbb{R}^{1} \rightarrow \mathbb{R}^{1}$ is a given continuous function, $a$ and $b$ are real constants, and $\alpha_{i}^{\prime} \mathrm{s}$ are positive real constants.

Motivated by the above mentioned works, we aim to establish the uniqueness of positive solutions for the fractional differential equation BVP (1.1). Different from the previous paper, the main features of the present paper are as follows. Firstly, the nonlinearity $f$ is allowed to depend on higher derivatives of unknown functions, and we allow $f$ to be singular at $t=0,1$ and $x_{i}=0(i=0,1, \ldots, n-2)$. Secondly, the fractional derivatives in the boundary conditions can be different; at the same time, the higher derivatives in the nonlinearity $f$ are also different from the fractional derivatives in the integral boundary conditions; and the boundary conditions of fractional differential BVP (1.1) contain [7, 9] as special cases. The boundary conditions involving fractional derivatives of unknown function are more general cases, which covers the multi-point boundary conditions and integral boundary conditions as special cases. Thirdly, the conclusion and the proof used in this paper are different from the known papers.

The paper is organized as follows. In Sect. 2, we present some preliminaries and lemmas that are to be used to prove our main results. We also develop some properties of the Green function. In Sect. 3, we discuss the existence of positive solutions of BVP (1.1). In Sect. 4, an example is presented to demonstrate the application of our main results.

\section{Preliminaries and lemmas}

In this section, for the convenience of the reader, we present some notations and lemmas that will be used in the proof of our main results. They can be found in the literature, see $[1,5,8,11,12]$.

Definition 2.1 $([1,5])$ The Riemann-Liouville fractional integral of order $\alpha>0$ of a function $y:(0, \infty) \rightarrow \mathbb{R}^{1}$ is given by

$$
I_{0^{+}}^{\alpha} y(t)=\frac{1}{\Gamma(\alpha)} \int_{0}^{t}(t-s)^{\alpha-1} y(s) d s
$$

provided that the right-hand side is pointwise defined on $(0, \infty)$.

Definition 2.2 $([1,5])$ The Riemann-Liouville fractional derivative of order $\alpha>0$ of a continuous function $y:(0, \infty) \rightarrow \mathbb{R}^{1}$ is given by

$$
D_{0^{+}}^{\alpha} y(t)=\frac{1}{\Gamma(n-\alpha)}\left(\frac{d}{d t}\right)^{n} \int_{0}^{t} \frac{y(s)}{(t-s)^{\alpha-n+1}} d s
$$

where $n=[\alpha]+1,[\alpha]$ denotes the integer part of the number $\alpha$, provided that the righthand side is pointwise defined on $(0, \infty)$.

Lemma 2.1 ([1]) Let $\alpha>0$. If we assume $u \in C(0,1) \cap L^{1}(0,1)$, then the fractional differential equation

$$
D_{0^{+}}^{\alpha} u(t)=0
$$


has

$$
u(t)=C_{1} t^{\alpha-1}+C_{2} t^{\alpha-2}+\cdots+C_{N} t^{\alpha-N},
$$

for some $C_{i} \in \mathbb{R}^{1}(i=1,2, \ldots, N)$, as the unique solution, where $N=[\alpha]+1$.

Lemma 2.2 ([1]) Assume that $u \in C(0,1) \cap L^{1}(0,1)$ with a fractional derivative of order $\alpha>0$ that belongs to $C(0,1) \cap L^{1}(0,1)$. Then

$$
I_{0^{+}}^{\alpha} D_{0^{+}}^{\alpha} u(t)=u(t)+C_{1} t^{\alpha-1}+C_{2} t^{\alpha-2}+\cdots+C_{N} t^{\alpha-N}
$$

for some $C_{i} \in \mathbb{R}^{1}(i=1,2, \ldots, N)$, where $N=[\alpha]+1$.

Lemma 2.3 ([1]) If $x \in L^{1}(0,1), \alpha>\beta>0$, then

$$
I_{0^{+}}^{\alpha} I_{0^{+}}^{\beta} x(t)=I_{0^{+}}^{\alpha+\beta} x(t), \quad D_{0^{+}}^{\beta} I_{0^{+}}^{\alpha} x(t)=I_{0^{+}}^{\alpha-\beta} x(t), \quad D_{0^{+}}^{\beta} I_{0^{+}}^{\beta} x(t)=x(t) .
$$

Lemma 2.4 (Auxiliary lemma) Given $y \in C(0,1) \cap L^{1}(0,1), n-1<\alpha \leq n$, and the following condition is satisfied:

$$
\text { (H) } \frac{\Gamma\left(\alpha-\beta_{1}\right)}{\Gamma\left(\alpha-\beta_{2}\right)} \int_{0}^{\eta} h(t) t^{\alpha-\beta_{2}-1} d A(t)+\frac{\Gamma\left(\alpha-\beta_{1}\right)}{\Gamma\left(\alpha-\beta_{3}\right)} \int_{0}^{1} a(t) t^{\alpha-\beta_{3}-1} d A(t)<1 \text {, }
$$

the unique solution of

$$
\left\{\begin{array}{l}
D_{0^{+}}^{\alpha-\alpha_{n-2}} x(t)+y(t)=0, \quad 0<t<1, \\
D_{0^{+}}^{\gamma_{n-2}-\alpha_{n-2}} x(0)=0, \\
D_{0^{+}}^{\beta_{1}-\alpha_{n-2}} x(1)=\int_{0}^{\eta} h(s) D_{0^{+}}^{\beta_{2}-\alpha_{n-2}} x(s) d A(s)+\int_{0}^{1} a(s) D_{0^{+}}^{\beta_{3}-\alpha_{n-2}} x(s) d A(s)
\end{array}\right.
$$

is

$$
x(t)=\int_{0}^{1} G(t, s) y(s) d s, \quad t \in[0,1]
$$

where

$$
\begin{aligned}
G(t, s)= & G_{1}(t, s)+\frac{t^{\alpha-\alpha_{n-2}-1}}{\Gamma\left(\alpha-\beta_{2}\right)} \Delta^{-1} \int_{0}^{\eta} h(t) G_{2}(t, s) d A(t) \\
& +\frac{t^{\alpha-\alpha_{n-2}-1}}{\Gamma\left(\alpha-\beta_{3}\right)} \Delta^{-1} \int_{0}^{1} a(t) G_{3}(t, s) d A(t),
\end{aligned}
$$


in which

$$
\begin{aligned}
& \delta_{1}:=\int_{0}^{\eta} h(t) t^{\alpha-\beta_{2}-1} d A(t), \quad \delta_{2}:=\int_{0}^{1} a(t) t^{\alpha-\beta_{3}-1} d A(t), \\
& \Delta=\frac{1}{\Gamma\left(\alpha-\beta_{1}\right)}-\frac{1}{\Gamma\left(\alpha-\beta_{2}\right)} \delta_{1}-\frac{1}{\Gamma\left(\alpha-\beta_{3}\right)} \delta_{2}>0, \\
& G_{1}(t, s)=\frac{1}{\Gamma\left(\alpha-\alpha_{n-2}\right)} \begin{cases}t^{\alpha-\alpha_{n-2}-1}(1-s)^{\alpha-\beta_{1}-1}-(t-s)^{\alpha-\alpha_{n-2}-1}, \\
0 \leq s \leq t \leq 1, \\
t^{\alpha-\alpha_{n-2}-1}(1-s)^{\alpha-\beta_{1}-1}, & 0 \leq t \leq s \leq 1,\end{cases} \\
& G_{2}(t, s)=\frac{1}{\Gamma\left(\alpha-\alpha_{n-2}\right)} \begin{cases}t^{\alpha-\beta_{2}-1}(1-s)^{\alpha-\beta_{1}-1}-(t-s)^{\alpha-\beta_{2}-1}, \\
t^{\alpha-\beta_{2}-1}(1-s)^{\alpha-\beta_{1}-1}, & 0 \leq t \leq s \leq 1 \leq 1,\end{cases} \\
& G_{3}(t, s)=\frac{1}{\Gamma\left(\alpha-\alpha_{n-2}\right)} \begin{cases}t^{\alpha-\beta_{3}-1}(1-s)^{\alpha-\beta_{1}-1}-(t-s)^{\alpha-\beta_{3}-1}, \\
t^{\alpha-\beta_{3}-1}(1-s)^{\alpha-\beta_{1}-1}, & 0 \leq t \leq s \leq 1 \leq 1,\end{cases}
\end{aligned}
$$

Proof We may apply Lemma 2.2 to reduce (2.1) to an equivalent integral equation

$$
x(t)=-I_{0^{+}}^{\alpha-\alpha_{n-2}} y(t)+C_{1} t^{\alpha-\alpha_{n-2}-1}+C_{2} t^{\alpha-\alpha_{n-2}-2}
$$

for some $C_{i} \in \mathbb{R}^{1}(i=1,2)$. Consequently, the general solution of $(2.1)$ is

$$
x(t)=-\frac{1}{\Gamma\left(\alpha-\alpha_{n-2}\right)} \int_{0}^{t}(t-s)^{\alpha-\alpha_{n-2}-1} y(s) d s+C_{1} t^{\alpha-\alpha_{n-2}-1}+C_{2} t^{\alpha-\alpha_{n-2}-2} .
$$

By (2.4) and Lemma 2.3, we have

$$
\begin{aligned}
& D_{0^{+}}^{\gamma_{n-2}-\alpha_{n-2}} x(t) \\
&=- D_{0^{+}}^{\gamma_{n-2}-\alpha_{n-2}} I_{0^{+}}^{\alpha-\alpha_{n-2}} y(t)+C_{1} D_{0^{+}}^{\gamma_{n-2}-\alpha_{n-2}} t^{\alpha-\alpha_{n-2}-1} \\
&+C_{2} D_{0^{+}}^{\gamma_{n-2}-\alpha_{n-2}} t^{\alpha-\alpha_{n-2}-2} \\
&=-I_{0^{+}}^{\alpha-\gamma_{n-2}} y(t)+C_{1} \frac{\Gamma\left(\alpha-\alpha_{n-2}\right)}{\Gamma\left(\alpha-\gamma_{n-2}\right)} t^{\alpha-\gamma_{n-2}-1}+C_{2} \frac{\Gamma\left(\alpha-\alpha_{n-2}-1\right)}{\Gamma\left(\alpha-\gamma_{n-2}-1\right)} t^{\alpha-\gamma_{n-2}-2} \\
&=-\int_{0}^{t} \frac{(t-s)^{\alpha-\gamma_{n-2}-1}}{\Gamma\left(\alpha-\gamma_{n-2}\right)} y(s) d s+C_{1} \frac{\Gamma\left(\alpha-\alpha_{n-2}\right)}{\Gamma\left(\alpha-\gamma_{n-2}\right)} t^{\alpha-\gamma_{n-2}-1} \\
&+C_{2} \frac{\Gamma\left(\alpha-\alpha_{n-2}-1\right)}{\Gamma\left(\alpha-\gamma_{n-2}-1\right)} t^{\alpha-\gamma_{n-2}-2} .
\end{aligned}
$$

Considering the fact that $D_{0^{+}}^{\gamma_{n-2}-\alpha_{n-2}} x(0)=0$, one gets that $C_{2}=0$ by (2.5). Then we obtain

$$
x(t)=-\frac{1}{\Gamma\left(\alpha-\alpha_{n-2}\right)} \int_{0}^{t}(t-s)^{\alpha-\alpha_{n-2}-1} y(s) d s+C_{1} t^{\alpha-\alpha_{n-2}-1} .
$$


By (2.6) and Lemma 2.3, we have

$$
\begin{aligned}
D_{0^{+}}^{\beta_{i}-\alpha_{n-2}} x(t) & =-D_{0^{+}}^{\beta_{i}-\alpha_{n-2}} I_{0^{+}}^{\alpha-\alpha_{n-2}} y(t)+C_{1} D_{0^{+}}^{\beta_{i}-\alpha_{n-2}} t^{\alpha-\alpha_{n-2}-1} \\
& =-I_{0^{+}}^{\alpha-\beta_{i}} y(t)+C_{1} \frac{\Gamma\left(\alpha-\alpha_{n-2}\right)}{\Gamma\left(\alpha-\beta_{i}\right)} t^{\alpha-\beta_{i}-1} \\
& =-\int_{0}^{t} \frac{(t-s)^{\alpha-\beta_{i}-1}}{\Gamma\left(\alpha-\beta_{i}\right)} y(s) d s+C_{1} \frac{\Gamma\left(\alpha-\alpha_{n-2}\right)}{\Gamma\left(\alpha-\beta_{i}\right)} t^{\alpha-\beta_{i}-1} .
\end{aligned}
$$

So, from (2.7), we have

$$
\begin{aligned}
& D_{0^{+}}^{\beta_{1}-\alpha_{n-2}} x(1)=-\int_{0}^{1} \frac{(1-s)^{\alpha-\beta_{1}-1}}{\Gamma\left(\alpha-\beta_{1}\right)} y(s) d s+C_{1} \frac{\Gamma\left(\alpha-\alpha_{n-2}\right)}{\Gamma\left(\alpha-\beta_{1}\right)}, \\
& D_{0^{+}}^{\beta_{2}-\alpha_{n-2}} x(t)=-\int_{0}^{t} \frac{(t-s)^{\alpha-\beta_{2}-1}}{\Gamma\left(\alpha-\beta_{2}\right)} y(s) d s+C_{1} \frac{\Gamma\left(\alpha-\alpha_{n-2}\right)}{\Gamma\left(\alpha-\beta_{2}\right)} t^{\alpha-\beta_{2}-1}, \\
& D_{0^{+}}^{\beta_{3}-\alpha_{n-2}} x(t)=-\int_{0}^{t} \frac{(t-s)^{\alpha-\beta_{3}-1}}{\Gamma\left(\alpha-\beta_{3}\right)} y(s) d s+C_{1} \frac{\Gamma\left(\alpha-\alpha_{n-2}\right)}{\Gamma\left(\alpha-\beta_{3}\right)} t^{\alpha-\beta_{3}-1} .
\end{aligned}
$$

On the other hand, by

$$
D_{0^{+}}^{\beta_{1}-\alpha_{n-2}} x(1)=\int_{0}^{\eta} h(s) D_{0^{+}}^{\beta_{2}-\alpha_{n-2}} x(s) d A(s)+\int_{0}^{1} a(s) D_{0^{+}}^{\beta_{3}-\alpha_{n-2}} x(s) d A(s)
$$

combining with (2.8), we obtain

$$
\begin{aligned}
C_{1}= & {\left[\Gamma\left(\alpha-\alpha_{n-2}\right) \Delta\right]^{-1}\left(\int_{0}^{1} \frac{(1-s)^{\alpha-\beta_{1}-1}}{\Gamma\left(\alpha-\beta_{1}\right)} y(s) d s-\int_{0}^{\eta} h(t) \int_{0}^{t} \frac{(t-s)^{\alpha-\beta_{2}-1}}{\Gamma\left(\alpha-\beta_{2}\right)} y(s) d s d A(t)\right.} \\
& \left.-\int_{0}^{1} a(t) \int_{0}^{t} \frac{(t-s)^{\alpha-\beta_{3}-1}}{\Gamma\left(\alpha-\beta_{3}\right)} y(s) d s d A(t)\right) .
\end{aligned}
$$

So, substituting $C_{1}$ into (2.6), one has the unique solution of problem (2.1):

$$
\begin{aligned}
x(t)= & \frac{1}{\Gamma\left(\alpha-\alpha_{n-2}\right)} \int_{0}^{t}(t-s)^{\alpha-\alpha_{n-2}-1} y(s) d s \\
& +t^{\alpha-\alpha_{n-2}-1}\left[\Gamma\left(\alpha-\alpha_{n-2}\right) \Delta\right]^{-1}\left(\int_{0}^{1} \frac{(1-s)^{\alpha-\beta_{1}-1}}{\Gamma\left(\alpha-\beta_{1}\right)} y(s) d s\right. \\
& \left.-\int_{0}^{\eta} h(t) \int_{0}^{t} \frac{(t-s)^{\alpha-\beta_{2}-1}}{\Gamma\left(\alpha-\beta_{2}\right)} y(s) d s d A(t)-\int_{0}^{1} a(t) \int_{0}^{t} \frac{(t-s)^{\alpha-\beta_{3}-1}}{\Gamma\left(\alpha-\beta_{3}\right)} y(s) d s d A(t)\right) \\
= & -\frac{1}{\Gamma\left(\alpha-\alpha_{n-2}\right)} \int_{0}^{t}(t-s)^{\alpha-\alpha_{n-2}-1} y(s) d s+\left[\frac{t^{\alpha-\alpha_{n-2}-1}}{\Gamma\left(\alpha-\alpha_{n-2}\right)}\right. \\
& \left.+\left(\Gamma\left(\alpha-\alpha_{n-2}\right) \Delta\right)^{-1}\left(\frac{\delta_{1} t^{\alpha-\alpha_{n-2}-1}}{\Gamma\left(\alpha-\beta_{2}\right)}+\frac{\delta_{2} t^{\alpha-\alpha_{n-2}-1}}{\Gamma\left(\alpha-\beta_{3}\right)}\right)\right] \int_{0}^{1}(1-s)^{\alpha-\beta_{1}-1} y(s) d s \\
& -\left[\Gamma\left(\alpha-\alpha_{n-2}\right) \Delta\right]^{-1} \frac{t^{\alpha-\alpha_{n-2}-1}}{\Gamma\left(\alpha-\beta_{2}\right)} \int_{0}^{\eta} h(t) \int_{0}^{t}(t-s)^{\alpha-\beta_{2}-1} y(s) d s \\
& -\left[\Gamma\left(\alpha-\alpha_{n-2}\right) \Delta\right]^{-1} \frac{t^{\alpha-\alpha_{n-2}-1}}{\Gamma\left(\alpha-\beta_{3}\right)} \int_{0}^{1} a(t) \int_{0}^{t}(t-s)^{\alpha-\beta_{3}-1} y(s) d s \\
= & \frac{1}{\Gamma\left(\alpha-\alpha_{n-2}\right)} \int_{0}^{t}(t-s)^{\alpha-\alpha_{n-2}-1} y(s) d s
\end{aligned}
$$




$$
\begin{aligned}
& +\frac{1}{\Gamma\left(\alpha-\alpha_{n-2}\right)} \int_{0}^{1} t^{\alpha-\alpha_{n-2}-1}(1-s)^{\alpha-\beta_{1}-1} y(s) d s \\
& +\left[\Gamma\left(\alpha-\alpha_{n-2}\right) \Delta\right]^{-1} \frac{t^{\alpha-\alpha_{n-2}-1}}{\Gamma\left(\alpha-\beta_{2}\right)} \int_{0}^{\eta} h(t) \int_{0}^{1} t^{\alpha-\beta_{2}-1}(1-s)^{\alpha-\beta_{1}-1} y(s) d s d A(t) \\
& +\left[\Gamma\left(\alpha-\alpha_{n-2}\right) \Delta\right]^{-1} \frac{t^{\alpha-\alpha_{n-2}-1}}{\Gamma\left(\alpha-\beta_{3}\right)} \int_{0}^{1} a(t) \int_{0}^{1} t^{\alpha-\beta_{3}-1}(1-s)^{\alpha-\beta_{1}-1} y(s) d s d A(t) \\
& -\left[\Gamma\left(\alpha-\alpha_{n-2}\right) \Delta\right]^{-1} \frac{t^{\alpha-\alpha_{n-2}-1}}{\Gamma\left(\alpha-\beta_{2}\right)} \int_{0}^{\eta} h(t) \int_{0}^{t}(t-s)^{\alpha-\beta_{2}-1} y(s) d s d A(t) \\
& -\left[\Gamma\left(\alpha-\alpha_{n-2}\right) \Delta\right]^{-1} \frac{t^{\alpha-\alpha_{n-2}-1}}{\Gamma\left(\alpha-\beta_{3}\right)} \int_{0}^{1} a(t) \int_{0}^{t}(t-s)^{\alpha-\beta_{3}-1} y(s) d s d A(t) \\
& =\int_{0}^{1}\left[G_{1}(t, s)+\Delta^{-1} \frac{t^{\alpha-\alpha_{n-2}-1}}{\Gamma\left(\alpha-\beta_{2}\right)} \int_{0}^{\eta} h(t) G_{2}(t, s) d A(t)\right. \\
& \left.+\Delta^{-1} \frac{t^{\alpha-\alpha_{n-2}-1}}{\Gamma\left(\alpha-\beta_{3}\right)} \int_{0}^{1} a(t) G_{3}(t, s) d A(t)\right] y(s) d s .
\end{aligned}
$$

The proof is complete.

Lemma 2.5 If the condition $(H)$ in Lemma 2.4 is satisfied, the Green function $G(t, s)$ defined by (2.3) satisfies

(1) $G(t, s)>0$, for all $t, s \in(0,1)$;

(2) $G_{1}(t, s) \leq \frac{t^{\alpha-\alpha_{n-2}-1}}{\Gamma\left(\alpha-\alpha_{n-2}\right)}, G_{2}(t, s) \leq \frac{1}{\Gamma\left(\alpha-\alpha_{n-2}\right)}, G_{3}(t, s) \leq \frac{1}{\Gamma\left(\alpha-\alpha_{n-2}\right)}$ for all $t, s \in[0,1]$;

(3) For any $t, s \in[0,1]$, we have

$$
t^{\alpha-\alpha_{n-2}-1} l_{1}(s) \leq G(t, s) \leq L_{1} t^{\alpha-\alpha_{n-2}-1},
$$

where

$$
\begin{aligned}
l_{1}(s)= & \frac{\Delta^{-1}}{\Gamma\left(\alpha-\beta_{2}\right)} \int_{0}^{\eta} h(t) G_{2}(t, s) d A(t) \\
& +\frac{\Delta^{-1}}{\Gamma\left(\alpha-\beta_{3}\right)} \int_{0}^{1} a(t) G_{3}(t, s) d A(t) \\
L_{1}= & \frac{1}{\Gamma\left(\alpha-\alpha_{n-2}\right)}+\frac{\Delta^{-1}}{\Gamma\left(\alpha-\beta_{2}\right)} \frac{1}{\Gamma\left(\alpha-\alpha_{n-2}\right)} \int_{0}^{\eta} h(t) d A(t) \\
& +\frac{\Delta^{-1}}{\Gamma\left(\alpha-\beta_{3}\right)} \frac{1}{\Gamma\left(\alpha-\alpha_{n-2}\right)} \int_{0}^{1} a(t) d A(t) .
\end{aligned}
$$

Proof The proof is similar to that for Lemma 3 in [19], we omit it here.

Let $u(t)=I_{0^{+}}^{\alpha_{n-2}} x(t), x(t) \in C[0,1]$, then $D_{0^{+}}^{\alpha_{n-2}} u(t)=x(t)$, problem (1.1) can turn into the following modified problem of BVP (2.9):

$$
\left\{\begin{array}{l}
D_{0^{+}}^{\alpha-\alpha_{n-2}} x(t)+f\left(t, I_{0^{+}}^{\alpha_{n-2}} x(t), I_{0^{+}}^{\alpha_{n-2}-\alpha_{1}} x(t), \ldots, x(t)\right)=0, \quad t \in(0,1) \\
D_{0^{+}}^{\gamma_{n-2}-\alpha_{n-2}} x(0)=0 \\
D_{0^{+}}^{\beta_{1}-\alpha_{n-2}} x(1)=\int_{0}^{\eta} h(s) D_{0^{+}}^{\beta_{2}-\alpha_{n-2}} x(s) d A(s)+\int_{0}^{1} a(s) D_{0^{+}}^{\beta_{3}-\alpha_{n-2}} x(s) d A(s) .
\end{array}\right.
$$


Obviously, the solution of BVP (2.9) is

$$
x(t)=\int_{0}^{1} G(t, s) f\left(s, I_{0^{+}}^{\alpha_{n-2}} x(s), I_{0^{+}}^{\alpha_{n-2}-\alpha_{1}} x(s), \ldots, x(s)\right) d s .
$$

Lemma 2.6 If $x \in C[0,1]$ is a positive solution of $B V P(2.9)$, let $u(t)=I_{0^{+}}^{\alpha_{n-2}} x(t), x(t) \in$ $C[0,1]$, then $u(t)=I_{0^{+}}^{\alpha_{n-2}} x(t)$ is a positive solution of $B V P(1.1)$.

Proof The proof is similar to that for Lemma 2.5 in [21], we omit it here.

Suppose that $(E,\|\cdot\|)$ is a Banach space and $\theta$ is the zero element of $E$. A nonempty closed convex set $P \subset E$ is a cone if it satisfies (1) $x \in P, \lambda \geq 0 \Rightarrow \lambda x \in P$; (2) $x \in P,-x \in$ $P \Rightarrow x=\theta$. Moreover, $P$ is called normal if there exists a constant $N>0$ such that, for all $x, y \in E, \theta \leq x \leq y$ implies $\|x\| \leq N\|y\|$, where the smallest $N$ is called the normality constant of $P$. Let $P$ be a normal cone of a Banach space $E$, and $e \in P, e>\theta$. Define a component of $P$ by $P_{e}=\{u \in P$ : there exist constants $c, C>0$ such that $c e \leq u \leq$ $\mathrm{Ce}$.

Definition 2.3 ([8]) $A: P \times P \rightarrow P$ is said to be a mixed monotone operator if $A(x, y)$ is increasing in $x$ and decreasing in $y$, i.e., $u_{i}, v_{i}(i=1,2) \in P, u_{1} \leq u_{2}, v_{1} \geq v_{2}$, imply $A\left(u_{1}, v_{1}\right) \leq A\left(u_{2}, v_{2}\right)$. An element $x \in P$ is called a fixed point of $A$ if $A(x, x)=$ $x$.

Lemma $2.7([10,24,28])$ Let $P$ be a normal cone in a real Banach space $E, e>0, P_{e}$ is a component of $P$. Assume that $A, B: P_{e} \times P_{e} \rightarrow P_{e}$ are two mixed monotone operators and satisfy the following three conditions:

(1) For any $t \in(0,1)$, there exists $\varphi(t) \in(0,1]$ such that

$$
A\left(t x, t^{-1} y\right) \geq \varphi(t) A(x, y), \quad \forall x, y \in P_{e} ;
$$

(2) For any $t \in(0,1), x, y \in P_{e}$,

$$
B\left(t x, t^{-1} y\right) \geq t B(x, y)
$$

(3) There exists a constant $\delta_{0}>0$ such that $B(x, y) \leq \delta_{0} A(x, y), \forall x, y \in P_{e}$. Then the operator equation $T(x, x) \triangleq A(x, x)+B(x, x)=x$ has a unique positive solution $x^{*}$ in $P$, which satisfies $c e \leq x^{*} \leq C e$, where $c, C>0$ are two real numbers. And for any initial values $x_{0}, y_{0} \in P_{e}$, by constructing successively the sequences as follows:

$$
x_{n}=T\left(x_{n-1}, y_{n-1}\right), \quad y_{n}=T\left(y_{n-1}, x_{n-1}\right), \quad n=1,2, \ldots,
$$

we have $x_{n} \rightarrow x^{*}$ and $y_{n} \rightarrow y^{*}$ in $E$, as $n \rightarrow \infty$.

Lemma $2.8([10,24,28])$ Let $P$ be a normal cone in a real Banach space E. Assume that $T=A+B: P_{e} \times P_{e} \rightarrow P_{e}$ is a mixed monotone operator and $A, B$ satisfy the conditions of Lemma 2.7 for $\varphi(t)=t^{\beta}(0<\beta<1)$. If $x_{\lambda}^{*} \in P_{e}$ is a unique solution of the equation

$$
T(x, x)=\lambda x, \quad \lambda>0,
$$


then

(1) For any $\lambda_{0} \in(0,+\infty],\left\|x_{\lambda}^{*}-x_{\lambda_{0}}^{*}\right\| \rightarrow 0, \lambda \rightarrow \lambda_{0}$.

(2) If $0<\beta<\frac{1}{2}$, then $0<\lambda_{1}<\lambda_{2}$ implies $x_{\lambda_{1}}^{*} \gg x_{\lambda_{2}}^{*}$ and $\lim _{\lambda \rightarrow \infty}\left\|x_{\lambda}^{*}\right\|=0$,

$\lim _{\lambda \rightarrow 0^{+}}\left\|x_{\lambda}^{*}\right\|=\infty$.

We here define a normal cone of the Banach space $E=C[0,1]$ by $P=\{u \in E: u(t) \geq$ $0,0 \leq t \leq 1\}$, and we also define a component $P_{e}=\left\{u \in P: \exists M \in(0,1), M e \leq u \leq \frac{1}{M} e\right\}$ of $P$, where $e(t)=t^{\alpha-\alpha_{n-2}-1} \in P, t \in[0,1]$.

\section{Uniqueness of a positive solution for BVP}

Theorem 3.1 The fractional BVP (1.1) has a unique positive solution $u^{*}$ with

$$
M \frac{\Gamma\left(\alpha-\alpha_{n-2}\right)}{\Gamma(\alpha)} t^{\alpha-1} \leq u^{*}(t) \leq \frac{1}{M} \frac{\Gamma\left(\alpha-\alpha_{n-2}\right)}{\Gamma(\alpha)} t^{\alpha-1}
$$

if the following conditions are satisfied:

$\left(H_{1}\right)$ For any $t \in(0,1)$ and $x_{i} \in(0,+\infty)(i=0,1,2, \ldots, n-2), f\left(t, x_{0}, x_{1}, \ldots, x_{n-2}\right)=$ $g\left(t, x_{0}, x_{1}, \ldots, x_{n-2} ; x_{0}, x_{1}, \ldots, x_{n-2}\right)+\phi\left(t, x_{0}, x_{1}, \ldots, x_{n-2} ; x_{0}, x_{1}, \ldots, x_{n-2}\right)$ with $g \in C((0$, 1) $\left.\times(0,+\infty)^{2(n-1)}, \mathbb{R}_{+}^{1}\right), \phi \in C\left((0,1) \times(0,+\infty)^{2(n-1)}, \mathbb{R}_{+}^{1}\right)$.

$\left(H_{2}\right)$ For any fixed $t \in(0,1)$ and $y_{i} \in(0,+\infty)(i=0,1,2, \ldots, n-2), g\left(t, x_{0}, x_{1}, \ldots, x_{n-2}, y_{0}, y_{1}\right.$, $\left.\ldots, y_{n-2}\right)$ and $\phi\left(t, x_{0}, x_{1}, \ldots, x_{n-2}, y_{0}, y_{1}, \ldots, y_{n-2}\right)$ are increasing in $x_{i} \in(0,+\infty)$, for any fixed $t \in(0,1)$ and $x_{i} \in(0,+\infty)(i=0,1,2, \ldots, n-2), g\left(t, x_{0}, x_{1}, \ldots, x_{n-2}, y_{0}, y_{1}, \ldots\right.$, $\left.y_{n-2}\right)$ and $\phi\left(t, x_{0}, x_{1}, \ldots, x_{n-2}, y_{0}, y_{1}, \ldots, y_{n-2}\right)$ are decreasing in $y_{i} \in(0,+\infty)$.

$\left(H_{3}\right)$ For any $\tau \in(0,1), t \in(0,1)$, there exists $\varphi(\tau) \in(\tau, 1]$ such that, for all $x_{i}, y_{i} \in$ $(0,+\infty)(i=0,1,2, \ldots, n-2)$,

$$
\begin{aligned}
& g\left(t, \tau x_{0}, \tau x_{1}, \ldots, \tau x_{n-2}, \tau^{-1} y_{0}, \tau^{-1} y_{1}, \ldots, \tau^{-1} y_{n-2}\right) \\
& \quad \geq \varphi(\tau) g\left(t, x_{0}, x_{1}, \ldots, x_{n-2}, y_{0}, y_{1}, \ldots, y_{n-2}\right), \\
& \phi\left(t, \tau x_{0}, \tau x_{1}, \ldots, \tau x_{n-2}, \tau^{-1} y_{0}, \tau^{-1} y_{1}, \ldots, \tau^{-1} y_{n-2}\right) \\
& \quad \geq \tau \phi\left(t, x_{0}, x_{1}, \ldots, x_{n-2}, y_{0}, y_{1}, \ldots, y_{n-2}\right) .
\end{aligned}
$$

$\left(H_{4}\right)$ For any fixed $t \in(0,1)$ and $x_{i}, y_{i} \in(0,+\infty)(i=0,1,2, \ldots, n-2)$, there exists a constant $\delta_{0}>0$ such that

$$
\phi\left(t, x_{0}, x_{1}, \ldots, x_{n-2}, y_{0}, y_{1}, \ldots, y_{n-2}\right) \leq \delta_{0} g\left(t, x_{0}, x_{1}, \ldots, x_{n-2}, y_{0}, y_{1}, \ldots, y_{n-2}\right) .
$$

$\left(H_{5}\right)$ The functions $g$ and $\phi$ satisfy

$$
\begin{aligned}
& 0<\int_{0}^{1} g\left(s, 1,1, \ldots, 1, s^{\alpha-1}, s^{\alpha-1}, \ldots, s^{\alpha-1}\right) d s<+\infty, \\
& 0<\int_{0}^{1} \phi\left(s, 1,1, \ldots, 1, s^{\alpha-1}, s^{\alpha-1}, \ldots, s^{\alpha-1}\right) d s<+\infty .
\end{aligned}
$$

Proof We first consider the existence of a positive solution to BVP (2.9), BVP (2.9) has an integral formulation given by

$$
x(t)=\int_{0}^{1} G(t, s) f\left(s, I_{0^{+}}^{\alpha_{n-2}} x(s), I_{0^{+}}^{\alpha_{n-2}-\alpha_{1}} x(s), \ldots, x(s)\right) d s,
$$


where $G(t, s)$ is given as in (2.2). Define three integral operators $A, B, T: P_{e} \times P_{e} \rightarrow P$ by

$$
\begin{aligned}
A(x, y)(t)= & \int_{0}^{1} G(t, s) g\left(s, I_{0^{+}}^{\alpha_{n-2}} x(s), I_{0^{+}}^{\alpha_{n-2}-\alpha_{1}} x(s), \ldots, x(s), I_{0^{+}}^{\alpha_{n-2}} y(s), I_{0^{+}}^{\alpha_{n-2}-\alpha_{1}} y(s),\right. \\
& \ldots, y(s)) d s, \\
B(x, y)(t)= & \int_{0}^{1} G(t, s) \phi\left(s, I_{0^{+}}^{\alpha_{n-2}} x(s), I_{0^{+}}^{\alpha_{n-2}-\alpha_{1}} x(s), \ldots, x(s), I_{0^{+}}^{\alpha_{n-2}} y(s), I_{0^{+}}^{\alpha_{n-2}-\alpha_{1}} y(s),\right. \\
& \ldots, y(s)) d s, \\
T(x, y)= & A(x, y)+B(x, y) .
\end{aligned}
$$

It is easy to prove that $x$ is the solution of BVP (2.9) if and only of $T$ in $P_{e}$ has a fixed point $x$, that is, $x=T(x, x)=A(x, x)+B(x, x)$.

First of all, we prove that $A, B: P_{e} \times P_{e} \rightarrow P$ are well defined. For any $x, y \in P_{e}$, there exists a constant $M \in(0,1)$ such that $M e \leq x \leq \frac{1}{M} e, M e \leq y \leq \frac{1}{M} e$. Moreover, by Definition 2.1, for any $t \in[0,1]$, we can obtain that

$$
\begin{aligned}
0 \leq I_{0^{+}}^{\alpha_{n-2}-\alpha_{i}} e(t) & =\frac{1}{\Gamma\left(\alpha_{n-2}-\alpha_{i}\right)} \int_{0}^{t}(t-s)^{\alpha_{n-2}-\alpha_{i}-1} s^{\alpha-\alpha_{n-2}-1} d s \\
& =\frac{B\left(\alpha_{n-2}-\alpha_{i}, \alpha-\alpha_{n-2}\right)}{\Gamma\left(\alpha_{n-2}-\alpha_{i}\right)} t^{\alpha-\alpha_{i}-1} \\
& =\frac{\Gamma\left(\alpha-\alpha_{n-2}\right)}{\Gamma\left(\alpha-\alpha_{i}\right)} t^{\alpha-\alpha_{i}-1} \leq 1, \quad i=0,1,2, \ldots, n-2 .
\end{aligned}
$$

Thus, by $\left(H_{2}\right),\left(H_{3}\right),\left(H_{5}\right)$ and (3) in Lemma 2.5 , for any $t \in[0,1]$, we have

$$
\begin{aligned}
A(x, y)(t) \leq & \int_{0}^{1} G(t, s) g\left(s, I_{0^{+}}^{\alpha_{n-2}} x(s), I_{0^{+}}^{\alpha_{n-2}-\alpha_{1}} x(s), \ldots, x(s), I_{0^{+}}^{\alpha_{n-2}} y(s)\right. \\
& \left.I_{0^{+}-\alpha^{-}-\alpha_{1}}^{\alpha} y(s), \ldots, y(s)\right) d s \\
\leq & \int_{0}^{1} G(t, s) g\left(s, I_{0^{+}}^{\alpha_{n-2}} M^{-1} e(s), I_{0^{+}}^{\alpha_{n-2}-\alpha_{1}} M^{-1} e(s), \ldots, M^{-1} e(s),\right. \\
& \left.I_{0^{+}}^{\alpha_{n-2}} M e(s), I_{0^{+}}^{\alpha_{n-2}-\alpha_{1}} M e(s), \ldots, M e(s)\right) d s \\
\leq & \int_{0}^{1} G(t, s) g\left(s, M^{-1}, M^{-1}, \ldots, M^{-1}, M \omega s^{\alpha-1}, M \omega s^{\alpha-\alpha_{1}-1}, \ldots,\right. \\
& \left.M \omega s^{\alpha-\alpha_{n-2}-1}\right) d s \\
\leq & \int_{0}^{1} G(t, s) g\left(s, \frac{1}{M \omega}, \frac{1}{M \omega}, \ldots, \frac{1}{M \omega}, M \omega s^{\alpha-1},\right. \\
& \left.M \omega s^{\alpha-1}, \ldots, M \omega s^{\alpha-1}\right) d s \\
\leq & \frac{1}{\varphi(M \omega)} L_{1} e(t) \int_{0}^{1} g\left(s, 1,1, \ldots, 1, s^{\alpha-1}, s^{\alpha-1}, \ldots, s^{\alpha-1}\right) d s \\
< & +\infty
\end{aligned}
$$


where

$$
0<\omega<\min \left\{1, \frac{\Gamma\left(\alpha-\alpha_{n-2}\right)}{\Gamma(\alpha)}, \frac{\Gamma\left(\alpha-\alpha_{n-2}\right)}{\Gamma\left(\alpha-\alpha_{1}\right)}, \ldots, \frac{\Gamma\left(\alpha-\alpha_{n-2}\right)}{\Gamma\left(\alpha-\alpha_{n-3}\right)}\right\} .
$$

Analogously, for any $t \in[0,1]$, we have

$$
\begin{aligned}
B(x, y)(t) & \leq \frac{1}{M \omega} L_{1} e(t) \int_{0}^{1} \phi\left(s, 1,1, \ldots, 1, s^{\alpha-1}, s^{\alpha-1}, \ldots, s^{\alpha-1}\right) d s \\
& <+\infty
\end{aligned}
$$

So, $A, B: P_{e} \times P_{e} \rightarrow P$ are well defined, therefore $T: P_{e} \times P_{e} \rightarrow P$ is well defined.

Next, we show $T: P_{e} \times P_{e} \rightarrow P_{e}$. But we first show that $A, B: P_{e} \times P_{e} \rightarrow P_{e}$. Taking $0<M<1$ satisfies

$$
\begin{aligned}
M< & \min \left\{\left(\frac{1}{\varphi(M \omega)} L_{1} \int_{0}^{1} g\left(s, 1,1, \ldots, 1, s^{\alpha-1}, s^{\alpha-1}, \ldots, s^{\alpha-1}\right) d s\right.\right. \\
& \left.+\frac{1}{M \omega} L_{1} \int_{0}^{1} \phi\left(s, 1,1, \ldots, 1, s^{\alpha-1}, s^{\alpha-1}, \ldots, s^{\alpha-1}\right) d s\right)^{-1}, \\
& \varphi(M \omega) \int_{0}^{1} l_{1}(s) g\left(s, s^{\alpha-1}, s^{\alpha-1}, \ldots, s^{\alpha-1}, 1,1, \ldots, 1\right) d s, \\
& \left.M \omega \int_{0}^{1} l_{1}(s) \phi\left(s, s^{\alpha-1}, s^{\alpha-1}, \ldots, s^{\alpha-1}, 1,1, \ldots, 1\right) d s\right\} .
\end{aligned}
$$

It follows from (3.1)-(3.3) that

$$
\begin{aligned}
A(x, y)(t) & \leq \frac{1}{\varphi(M \omega)} L_{1} t^{\alpha-\alpha_{n-2}-1} \int_{0}^{1} g\left(s, 1,1, \ldots, 1, s^{\alpha-1}, s^{\alpha-1}, \ldots, s^{\alpha-1}\right) d s \\
& \leq M^{-1} t^{\alpha-\alpha_{n-2}-1} \\
& =M^{-1} e(t), \quad t \in[0,1] \\
B(x, y)(t) & \leq \frac{1}{M \omega} L_{1} t^{\alpha-\alpha_{n-2}-1} \int_{0}^{1} \phi\left(s, 1,1, \ldots, 1, s^{\alpha-1}, s^{\alpha-1}, \ldots, s^{\alpha-1}\right) d s \\
& \leq M^{-1} t^{\alpha-\alpha_{n-2}-1} \\
& =M^{-1} e(t), \quad t \in[0,1] .
\end{aligned}
$$

Hence,

$$
\begin{aligned}
T(x, y)(t) \leq & \frac{1}{\varphi(M \omega)} L_{1} t^{\alpha-\alpha_{n-2}-1} \int_{0}^{1} g\left(s, 1,1, \ldots, 1, s^{\alpha-1}, s^{\alpha-1}, \ldots, s^{\alpha-1}\right) d s \\
& +\frac{1}{M \omega} L_{1} t^{\alpha-\alpha_{n-2}-1} \int_{0}^{1} \phi\left(s, 1,1, \ldots, 1, s^{\alpha-1}, s^{\alpha-1}, \ldots, s^{\alpha-1}\right) d s \\
\leq & M^{-1} t^{\alpha-\alpha_{n-2}-1} \\
= & M^{-1} e(t), \quad t \in[0,1] .
\end{aligned}
$$


On the other hand, by $\left(H_{2}\right),\left(H_{3}\right),(3.3)$, and (3) in Lemma 2.5 , for any $t \in[0,1]$, we have

$$
\begin{aligned}
& A(x, y)(t)=\int_{0}^{1} G(t, s) g\left(s, I_{0^{+}}^{\alpha_{n-2}} x(s), I_{0^{+}}^{\alpha_{n-2}-\alpha_{1}} x(s), \ldots, x(s), I_{0^{+}}^{\alpha_{n-2}} y(s), I_{0^{+}}^{\alpha_{n-2}-\alpha_{1}} y(s),\right. \\
& \ldots, y(s)) d s \\
& \geq \int_{0}^{1} G(t, s) g\left(s, I_{0^{+}}^{\alpha_{n-2}} M e(s), I_{0^{+}}^{\alpha_{n-2}-\alpha_{1}} M e(s), \ldots, M e(s), I_{0^{+}}^{\alpha_{n-2}} \frac{1}{M} e(s),\right. \\
& \left.I_{0^{+}}^{\alpha_{n-2}-\alpha_{1}} \frac{1}{M} e(s), \ldots, \frac{1}{M} e(s)\right) d s \\
& \geq \int_{0}^{1} G(t, s) g\left(s, M \omega s^{\alpha-1}, M \omega s^{\alpha-\alpha_{1}-1}, \ldots, M \omega s^{\alpha-\alpha_{n-2}-1}, \frac{1}{M \omega} s^{\alpha-\alpha_{n-2}-1},\right. \\
& \left.\frac{1}{M \omega} s^{\alpha-\alpha_{n-2}-1}, \ldots, \frac{1}{M \omega} s^{\alpha-\alpha_{n-2}-1}\right) d s \\
& \geq \int_{0}^{1} G(t, s) g\left(s, M \omega s^{\alpha-1}, M \omega s^{\alpha-1}, \ldots, M \omega s^{\alpha-1}, \frac{1}{M \omega} s^{\alpha-\alpha_{n-2}-1},\right. \\
& \left.\frac{1}{M \omega} s^{\alpha-\alpha_{n-2}-1}, \ldots, \frac{1}{M \omega} s^{\alpha-\alpha_{n-2}-1}\right) d s \\
& \geq \varphi(M \omega) t^{\alpha-\alpha_{n-2}-1} \int_{0}^{1} l_{1}(s) g\left(s, s^{\alpha-1}, s^{\alpha-1}, \ldots, s^{\alpha-1}, 1,1, \ldots, 1\right) d s \\
& \geq M t^{\alpha-\alpha_{n-2}-1} \\
& =M e(t) \text {. }
\end{aligned}
$$

Similarly, for any $t \in[0,1]$, we have

$$
\begin{aligned}
B(x, y)(t) & \geq M \omega t^{\alpha-\alpha_{n-2}-1} \int_{0}^{1} l_{1}(s) \phi\left(s, s^{\alpha-1}, s^{\alpha-1}, \ldots, s^{\alpha-1}, 1,1, \ldots, 1\right) d s \\
& \geq M t^{\alpha-\alpha_{n-2}-1} \\
& =M e(t) .
\end{aligned}
$$

Hence,

$$
\begin{aligned}
T(x, y)(t) \geq & \varphi(M \omega) t^{\alpha-\alpha_{n-2}-1} \int_{0}^{1} l_{1}(s) g\left(s, s^{\alpha-1}, s^{\alpha-1}, \ldots, s^{\alpha-1}, 1,1, \ldots, 1\right) d s \\
& +M \omega t^{\alpha-\alpha_{n-2}-1} \int_{0}^{1} l_{1}(s) \phi\left(s, s^{\alpha-1}, s^{\alpha-1}, \ldots, s^{\alpha-1}, 1,1, \ldots, 1\right) d s \\
\geq & M t^{\alpha-\alpha_{n-2}-1} \\
= & M e(t) .
\end{aligned}
$$

So, $A, B, T: P_{e} \times P_{e} \rightarrow P_{e}$.

In the following, we prove that $T, A, B: P_{e} \times P_{e} \rightarrow P_{e}$ are three mixed monotone operators. In fact, for any $x_{i}, y_{i} \in P_{e}(i=1,2)$ with $x_{1} \leq x_{2}, y_{1} \geq y_{2}$, from the monotonicity of 
$I^{i}(i>0)$ and $\left(H_{2}\right)$, we obtain

$$
\begin{aligned}
& \int_{0}^{1} G(t, s) g\left(s, I_{0^{+}}^{\alpha_{n-2}} x_{1}(s), I_{0^{+}}^{\alpha_{n-2}-\alpha_{1}} x_{1}(s), \ldots, x_{1}(s), I_{0^{+}}^{\alpha_{n-2}} y(s), I_{0^{+}}^{\alpha_{n-2}-\alpha_{1}} y(s), \ldots, y(s)\right) d s \\
& \quad \leq \int_{0}^{1} G(t, s) g\left(s, I_{0^{+}}^{\alpha_{n-2}} x_{2}(s), I_{0^{+}}^{\alpha_{n-2}-\alpha_{1}} x_{2}(s), \ldots, x_{2}(s), I_{0^{+}}^{\alpha_{n-2}} y(s), I_{0^{+}}^{\alpha_{n-2}-\alpha_{1}} y(s), \ldots,\right. \\
& \quad y(s)) d s, \\
& \int_{0}^{1} G(t, s) g\left(s, I_{0^{+}}^{\alpha_{n-2}} x(s), I_{0^{+}}^{\alpha_{n-2}-\alpha_{1}} x(s), \ldots, x(s), I_{0^{+}}^{\alpha_{n-2}} y_{1}(s), I_{0^{+}}^{\alpha_{n-2}-\alpha_{1}} y_{1}(s), \ldots, y_{1}(s)\right) d s \\
& \leq \int_{0}^{1} G(t, s) g\left(s, I_{0^{+}}^{\alpha_{n-2}} x(s), I_{0^{+}}^{\alpha_{n-2}-\alpha_{1}} x(s), \ldots, x(s), I_{0^{+}}^{\alpha_{n-2}} y_{2}(s), I_{0^{+}}^{\alpha_{n-2}-\alpha_{1}} y_{2}(s), \ldots,\right. \\
& \left.\quad y_{2}(s)\right) d s,
\end{aligned}
$$

which implies that

$$
\begin{aligned}
& A\left(x_{1}, y\right)(t) \leq A\left(x_{2}, y\right)(t), \quad y \in P_{e}, t \in[0,1], \\
& A\left(x, y_{1}\right)(t) \leq A\left(x, y_{2}\right)(t), \quad x \in P_{e}, t \in[0,1],
\end{aligned}
$$

that is, $A(x, y)$ is nondecreasing in $x$ for any $y \in P_{e}, A(x, y)$ is nonincreasing in $y$ for any $x \in P_{e}$. Similarly, we obtain

$$
\begin{array}{ll}
B\left(x_{1}, y\right)(t) \leq B\left(x_{2}, y\right)(t), & y \in P_{e}, t \in[0,1], \\
B\left(x, y_{1}\right)(t) \leq B\left(x, y_{2}\right)(t), & x \in P_{e}, t \in[0,1],
\end{array}
$$

that is, $B(x, y)$ is nondecreasing in $x$ for any $y \in P_{e}, B(x, y)$ is nonincreasing in $y$ for any $x \in P_{e}$. Hence, the operators $A, B$ are two mixed monotone operators, therefore $T$ is a mixed monotone operator.

For any $\tau \in(0,1)$ and $x, y \in P_{e}$, by $\left(H_{3}\right)$, we obtain

$$
\begin{aligned}
A\left(\tau x, \tau^{-1} y\right)(t)= & \int_{0}^{1} G(t, s) g\left(s, I_{0^{+}}^{\alpha_{n-2}} \tau x(s), I_{0^{+}}^{\alpha_{n-2}-\alpha_{1}} \tau x(s), \ldots, \tau x(s), I_{0^{+}}^{\alpha_{n-2}} \tau^{-1} y(s),\right. \\
& \left.I_{0^{+}}^{\alpha_{n-2}-\alpha_{1}} \tau^{-1} y(s), \ldots, \tau^{-1} y(s)\right) d s \\
= & \int_{0}^{1} G(t, s) g\left(s, \tau I_{0^{+}}^{\alpha_{n-2}} x(s), \tau I_{0^{+}}^{\alpha_{n-2}-\alpha_{1}} x(s), \ldots, \tau x(s), \tau^{-1} I_{0^{+}}^{\alpha_{n-2}} y(s),\right. \\
& \left.\tau^{-1} I_{0^{+}}^{\alpha_{n-2}-\alpha_{1}} y(s), \ldots, \tau^{-1} y(s)\right) d s \\
\geq & \varphi(\tau) \int_{0}^{1} G(t, s) g\left(s, I_{0^{+}}^{\alpha_{n-2}} x(s), I_{0^{+}}^{\alpha_{n-2}-\alpha_{1}} x(s), \ldots, x(s), I_{0^{+}}^{\alpha_{n-2}} y(s),\right. \\
& \left.I_{0^{+}}^{\alpha_{n-2}-\alpha_{1}} y(s), \ldots, y(s)\right) d s \\
= & \varphi(\tau) A(x, y)(t), \quad t \in[0,1] .
\end{aligned}
$$


That is, $A\left(\tau x, \tau^{-1} y\right) \geq \varphi(\tau) A(x, y)$ for any $\tau \in(0,1), x, y \in P_{e}$. Also, for any $\tau \in(0,1)$ and $x, y \in P_{e}$, by $\left(H_{3}\right)$, we obtain

$$
\begin{aligned}
B\left(\tau x, \tau^{-1} y\right)(t) & \\
= & \int_{0}^{1} G(t, s) \phi\left(s, I_{0^{+}}^{\alpha_{n-2}} \tau x(s), I_{0^{+}}^{\alpha_{n-2}-\alpha_{1}} \tau x(s), \ldots, \tau x(s), I_{0^{+}}^{\alpha_{n-2}} \tau^{-1} y(s),\right. \\
& \left.I_{0^{+}}^{\alpha_{n-2}-\alpha_{1}} \tau^{-1} y(s), \ldots, \tau^{-1} y(s)\right) d s \\
= & \int_{0}^{1} G(t, s) \phi\left(s, \tau I_{0^{+}}^{\alpha_{n-2}} x(s), \tau I_{0^{+}}^{\alpha_{n-2}-\alpha_{1}} x(s), \ldots, \tau x(s), \tau^{-1} I_{0^{+}}^{\alpha_{n-2}} y(s),\right. \\
& \left.\tau^{-1} I_{0^{+}}^{\alpha_{n-2}-\alpha_{1}} y(s), \ldots, \tau^{-1} y(s)\right) d s \\
\geq & \tau \int_{0}^{1} G(t, s) \phi\left(s, I_{0^{+}}^{\alpha_{n-2}} x(s), I_{0^{+}}^{\alpha_{n-2}-\alpha_{1}} x(s), \ldots, x(s), I_{0^{+}}^{\alpha_{n-2}} y(s),\right. \\
& \left.I_{0^{+}}^{\alpha_{n-2}-\alpha_{1}} y(s), \ldots, y(s)\right) d s \\
= & \tau B(x, y)(t), \quad t \in[0,1] .
\end{aligned}
$$

That is, $B\left(\tau x, \tau^{-1} y\right) \geq \tau B(x, y)$ for any $\tau \in(0,1), x, y \in P_{e}$. Finally, we shall prove that $B(x, y) \leq \delta_{0} A(x, y)$ for all $x, y \in P_{e}$. For $x, y \in P_{e}$, from $\left(H_{4}\right)$, we have

$$
\begin{aligned}
B(x, y)(t)= & \int_{0}^{1} G(t, s) \phi\left(s, I_{0^{+}}^{\alpha_{n-2}} x(s), I_{0^{+}}^{\alpha_{n-2}-\alpha_{1}} x(s), \ldots, x(s), I_{0^{+}}^{\alpha_{n-2}} y(s),\right. \\
& \left.I_{0^{+}}^{\alpha_{n-2}-\alpha_{1}} y(s), \ldots, y(s)\right) d s \\
\leq & \int_{0}^{1} G(t, s) \delta_{0} g\left(s, I_{0^{+}}^{\alpha_{n-2}} x(s), I_{0^{+}}^{\alpha_{n-2}-\alpha_{1}} x(s), \ldots, x(s), I_{0^{+}}^{\alpha_{n-2}} y(s),\right. \\
& \left.I_{0^{+}}^{\alpha_{n-2}-\alpha_{1}} y(s), \ldots, y(s)\right) d s \\
= & \delta_{0} A(x, y)(t), \quad t \in[0,1] .
\end{aligned}
$$

Then we get

$$
B(x, y) \leq \delta_{0} A(x, y), \quad \forall x, y \in P_{e} .
$$

Thus, Lemma 2.7 ensures that there exists a unique positive solution $x^{*} \in P_{e}$ such that $T\left(x^{*}, x^{*}\right)=x^{*}$. Consequently, problem (2.9) has a unique positive solution $x^{*} \in P_{e}$. By Lemma 2.6, then $u^{*}(t)=I_{0^{+}-2}^{\alpha_{n-2}}(t)$ is the unique positive solution of BVP (1.1). Moreover,

$$
M \frac{\Gamma\left(\alpha-\alpha_{n-2}\right)}{\Gamma(\alpha)} t^{\alpha-1} \leq u^{*}(t)=I_{0^{+}}^{\alpha_{n-2}} x^{*}(t) \leq \frac{1}{M} \frac{\Gamma\left(\alpha-\alpha_{n-2}\right)}{\Gamma(\alpha)} t^{\alpha-1} .
$$

Therefore, the proof of Theorem 3.1 is completed.

Theorem 3.2 Assume that the conditions of Theorem 3.1 are satisfied for $\varphi(t)=t^{\beta}(0<\beta<$ 1), then BVP (1.1) has a unique solution $u_{\lambda}^{*}$ with

$$
M \frac{\Gamma\left(\alpha-\alpha_{n-2}\right)}{\Gamma(\alpha)} t^{\alpha-1} \leq u_{\lambda}^{*}(t) \leq \frac{1}{M} \frac{\Gamma\left(\alpha-\alpha_{n-2}\right)}{\Gamma(\alpha)} t^{\alpha-1}
$$


Furthermore, we have the following conclusions:

(1) For any $\lambda_{0} \in(0,+\infty],\left\|u_{\lambda}^{*}-u_{\lambda_{0}}^{*}\right\| \rightarrow 0, \lambda \rightarrow \lambda_{0}$.

(2) If $0<\beta<\frac{1}{2}$, then $0<\lambda_{1}<\lambda_{2}$ implies $u_{\lambda_{1}}^{*} \gg u_{\lambda_{2}}^{*}$ and $\lim _{\lambda \rightarrow \infty}\left\|u_{\lambda}^{*}\right\|=0$, $\lim _{\lambda \rightarrow 0^{+}}\left\|u_{\lambda}^{*}\right\|=+\infty$.

Proof For fixed $\lambda>0$, by Theorem 3.1, $\frac{1}{\lambda} A, \frac{1}{\lambda} B: P_{e} \times P_{e} \rightarrow P_{e}$ are two mixed monotone operators satisfying

$$
\begin{aligned}
& \frac{1}{\lambda} A\left(\tau x, \tau^{-1} y\right) \geq \varphi(\tau) \frac{1}{\lambda} A(x, y), \quad \varphi(t) \in(0,1], \\
& \frac{1}{\lambda} B\left(\tau x, \tau^{-1} y\right) \geq \tau \frac{1}{\lambda} B(x, y)
\end{aligned}
$$

for all $\tau \in(0,1), x, y \in P_{e}$. So it follows from the proof of Lemma 2.7 and Theorem 3.1 that $\frac{1}{\lambda} T: P_{e} \times P_{e} \rightarrow P_{e}$ is mixed monotone and satisfies

$$
\frac{1}{\lambda} T\left(\tau x, \tau^{-1} y\right) \geq \frac{1}{\lambda} \psi(\tau) T(x, y), \quad \varphi(t) \in(0,1] .
$$

Hence, it follows from Theorem 3.1 that $\frac{1}{\lambda} T$ has a unique fixed point $x_{\lambda}^{*} \in P_{e}$. That is, $T\left(x_{\lambda}^{*}, x_{\lambda}^{*}\right)=\lambda x_{\lambda}^{*}$. Moreover, by Lemma 2.8 we have the following conclusions:

(1) For any $\lambda_{0} \in(0,+\infty],\left\|x_{\lambda}^{*}-x_{\lambda_{0}}^{*}\right\| \rightarrow 0, \lambda \rightarrow \lambda_{0}$.

(2) If $0<\beta<\frac{1}{2}$, then $0<\lambda_{1}<\lambda_{2}$ implies $x_{\lambda_{1}}^{*} \gg x_{\lambda_{2}}^{*}$ and $\lim _{\lambda \rightarrow \infty}\left\|x_{\lambda}^{*}\right\|=0, \lim _{\lambda \rightarrow 0^{+}}\left\|x_{\lambda}^{*}\right\|=+\infty$.

By Lemma 2.6, we have $u_{\lambda}^{*}(t)=I_{0^{+}-2}^{\alpha_{n-2}^{*}}(t)$. Hence, by the monotonicity and continuity of $I_{0^{+}}^{\alpha_{n-2}}$, we get

(1) For any $\lambda_{0} \in(0,+\infty],\left\|u_{\lambda}^{*}-u_{\lambda_{0}}^{*}\right\| \rightarrow 0, \lambda \rightarrow \lambda_{0}$.

(2) If $0<\beta<\frac{1}{2}$, then $0<\lambda_{1}<\lambda_{2}$ implies $u_{\lambda_{1}}^{*} \gg u_{\lambda_{2}}^{*}$ and $\lim _{\lambda \rightarrow \infty}\left\|u_{\lambda}^{*}\right\|=0, \lim _{\lambda \rightarrow 0^{+}}\left\|u_{\lambda}^{*}\right\|=+\infty$.

Therefore, the proof of Theorem 3.2 is completed.

\section{An example}

Example 4.1 We consider the following boundary value problem:

$$
\left\{\begin{array}{l}
D_{0^{+}}^{\frac{5}{2}} u(t)+f\left(t, u(t), D_{0^{+}}^{\frac{1}{4}} u(t)\right)=0, \quad t \in(0,1), \\
u(0)=D_{0^{+}}^{\frac{3}{4}} u(0)=0, \quad u^{\prime}(1)=\int_{0}^{\eta} D_{0^{+}}^{\frac{5}{8}} u(s) d A(s)+\int_{0}^{1} D_{0^{+}}^{\frac{7}{8}} u(s) d A(s),
\end{array}\right.
$$

where $\alpha=\frac{5}{2}, a(s)=s^{\frac{9}{8}}, h(s)=1, \eta=1$, and

$$
A(t)= \begin{cases}0, & t \in\left[0, \frac{1}{2}\right) \\ \frac{1}{100}, & t \in\left[\frac{1}{2}, 1\right] .\end{cases}
$$

Let $u(t)=I_{0^{+}}^{\frac{1}{4}} x(t)$, equation (4.1) can be changed to the following:

$$
\left\{\begin{array}{l}
D_{0^{+}}^{\frac{9}{4}} x(t)+f\left(t, I_{0^{+}}^{\frac{1}{4}} x(t), x(t)\right)=0, \quad t \in(0,1), \\
D_{0^{+}}^{\frac{1}{2}} x(0)=0, \quad D_{0^{+}}^{\frac{3}{4}} x(1)=\int_{0}^{\eta} D_{0^{+}}^{\frac{3}{8}} x(s) d A(s)+\int_{0}^{1} D_{0^{+}}^{\frac{5}{8}} x(s) d A(s),
\end{array}\right.
$$


where

$$
\begin{aligned}
& f(t, x, y)=(1-t)^{-\frac{1}{3}} t^{-\frac{2}{3}} x^{\frac{1}{3}}+t^{-\frac{1}{2}} x^{\frac{1}{2}}+(t y)^{-\frac{1}{6}}+y^{-\frac{1}{5}}+(1-t)^{-\frac{1}{8}} t^{-\frac{1}{6}} x^{\frac{1}{3}} \\
& +x^{\frac{1}{2}}+y^{-\frac{1}{5}}+(2 y)^{-\frac{1}{6}} \\
& g(t, x, x, y, y)=(1-t)^{-\frac{1}{3}} t^{-\frac{2}{3}} x^{\frac{1}{3}}+t^{-\frac{1}{2}} x^{\frac{1}{2}}+(t y)^{-\frac{1}{6}}+y^{-\frac{1}{5}}, \\
& \phi(t, x, x, y, y)=(1-t)^{-\frac{1}{8}} t^{-\frac{1}{6}} x^{\frac{1}{3}}+x^{\frac{1}{2}}+y^{-\frac{1}{5}}+(2 y)^{-\frac{1}{6}}
\end{aligned}
$$

for any $t \in(0,1), x, y>0, f(t, x, y)=g(t, x, x, y, y)+\phi(t, x, x, y, y), e(t)=t^{\frac{5}{4}}$.

Let us check that all the required conditions of Theorem 3.1 are satisfied.

(1) Clearly, the functions $g, \phi:(0,1) \times(0,+\infty)^{4} \rightarrow[0,+\infty)$ are continuous.

(2) We observe easily that for fixed $t \in(0,1)$ and $y_{i} \in(0,+\infty)(i=1,2), g\left(t, x_{1}, x_{2}, y_{1}, y_{2}\right)$ is increasing in $x_{i} \in(0,+\infty)(i=1,2)$; for fixed $t \in(0,1)$ and $x_{i} \in(0,+\infty)(i=1,2)$, $\phi\left(t, x_{1}, x_{2}, y_{1}, y_{2}\right)$ is decreasing in $y_{i} \in(0,+\infty)(i=1,2)$.

(3) For all $\lambda \in(0,1), t \in(0,1)$, and $x_{i}, y_{i}>0(i=1,2)$, taking $\varphi(\lambda)=\lambda^{\frac{1}{2}} \in(\lambda, 1)$, we have

$$
\begin{aligned}
g( & \left.t, \lambda x_{1}, \lambda x_{2}, \lambda^{-1} y_{1}, \lambda^{-1} y_{2}\right) \\
& =(1-t)^{-\frac{1}{3}} t^{-\frac{2}{3}}\left(\lambda x_{1}\right)^{\frac{1}{3}}+t^{-\frac{1}{2}}\left(\lambda x_{2}\right)^{\frac{1}{2}}+\left(t \lambda^{-1} y_{1}\right)^{-\frac{1}{6}}+\left(\lambda^{-1} y_{2}\right)^{-\frac{1}{5}} \\
& =(1-t)^{-\frac{1}{3}} t^{-\frac{2}{3}} \lambda^{\frac{1}{3}}\left(x_{1}\right)^{\frac{1}{3}}+t^{-\frac{1}{2}} \lambda^{\frac{1}{2}}\left(x_{2}\right)^{\frac{1}{2}}+\lambda^{\frac{1}{6}}\left(t y_{1}\right)^{-\frac{1}{6}}+\lambda^{\frac{1}{5}}\left(y_{2}\right)^{-\frac{1}{5}} \\
& \geq \lambda^{\frac{1}{2}}\left[(1-t)^{-\frac{1}{3}} t^{-\frac{2}{3}} x_{1}^{\frac{1}{3}}+t^{-\frac{1}{2}} x_{2}^{\frac{1}{2}}+\left(t y_{1}\right)^{-\frac{1}{6}}+y_{2}^{-\frac{1}{5}}\right] \\
& =\varphi(\lambda) g\left(t, x_{1}, x_{2}, y_{1}, y_{2}\right) .
\end{aligned}
$$

For all $\lambda \in(0,1), t \in(0,1)$, and $x_{i}, y_{i}>0(i=1,2)$, we have

$$
\begin{aligned}
\phi( & \left.t, \lambda x_{1}, \lambda x_{2}, \lambda^{-1} y_{1}, \lambda^{-1} y_{2}\right) \\
& =(1-t)^{-\frac{1}{8}} t^{-\frac{1}{6}}\left(\lambda x_{1}\right)^{\frac{1}{3}}+\left(\lambda x_{2}\right)^{\frac{1}{2}}+\left(\lambda^{-1} y_{1}\right)^{-\frac{1}{5}}+\left(2 \lambda^{-1} y_{2}\right)^{-\frac{1}{6}} \\
& =(1-t)^{-\frac{1}{8}} t^{-\frac{1}{6}} \lambda^{\frac{1}{3}}\left(x_{1}\right)^{\frac{1}{3}}+\lambda^{\frac{1}{5}}\left(x_{2}\right)^{\frac{1}{2}}+\lambda^{\frac{1}{2}}\left(y_{1}\right)^{-\frac{1}{5}}+\lambda^{\frac{1}{6}}\left(2 y_{2}\right)^{-\frac{1}{6}} \\
& \geq \lambda\left[(1-t)^{-\frac{1}{8}} t^{-\frac{1}{6}} x^{\frac{1}{3}}+x^{\frac{1}{2}}+y^{-\frac{1}{5}}+(2 y)^{-\frac{1}{6}}\right] \\
& =\lambda \phi\left(t, x_{1}, x_{2}, y_{1}, y_{2}\right) .
\end{aligned}
$$

(4) Taking $\delta_{0}=2>0$, then

$$
\begin{aligned}
\phi\left(t, x_{1}, x_{2}, y_{1}, y_{2}\right) & =(1-t)^{-\frac{1}{8}} t^{-\frac{1}{6}} x^{\frac{1}{3}}+x^{\frac{1}{2}}+y^{-\frac{1}{5}}+(2 y)^{-\frac{1}{6}} \\
& \leq 2\left[(1-t)^{-\frac{1}{3}} t^{-\frac{2}{3}} x^{\frac{1}{3}}+t^{-\frac{1}{2}} x^{\frac{1}{2}}+(t y)^{-\frac{1}{6}}+y^{-\frac{1}{5}}\right] \\
& =\delta_{0} f\left(t, x_{1}, x_{2}, y_{1}, y_{2}\right) .
\end{aligned}
$$

(5)

$$
\int_{0}^{1} g\left(s, 1,1, s^{\frac{3}{2}}, s^{\frac{3}{2}}\right) d s=\int_{0}^{1}\left((1-s)^{-\frac{1}{3}} s^{-\frac{2}{3}}+s^{-\frac{1}{2}}+s^{-\frac{5}{12}}+s^{-\frac{3}{10}}\right) d s<+\infty .
$$




$$
\begin{aligned}
& \int_{0}^{1} \phi\left(s, 1,1, s^{\frac{3}{2}}, s^{\frac{3}{2}}\right) d s \\
& \quad=\int_{0}^{1}\left((1-s)^{-\frac{1}{8}} s^{-\frac{1}{6}}+1+s^{-\frac{1}{2}}+s^{-\frac{3}{10}}+2^{-\frac{1}{6}} s^{-\frac{1}{4}}\right) d s \\
& \quad<+\infty .
\end{aligned}
$$

Therefore the assumptions of Theorem 3.1 are satisfied. Then Theorem 3.1 implies that BVP (4.1) has a unique positive solution.

\section{Conclusions}

In this paper, by using the fixed point theorem of mixed monotone operator in cone, we have established the uniqueness of positive solutions for a class of singular fractional differential equations with integral boundary conditions. Fractional differential equations arise in the mathematical modeling of systems and processes occurring in many engineering and scientific disciplines such as polymer rheology, electrodynamics of complex medium. Moreover, fractional differential equations and integral operators are found to be a better tool for the description of hereditary properties of various materials and processes than the corresponding integer-order differential equations. The main contribution is that we divide the function into the form of $g+\phi$ and add different conditions to $g$ and $\phi$. Furthermore, the higher derivatives in the nonlinearity $f$ are also different from the fractional derivatives in the integral boundary conditions, and boundary conditions of fractional differential BVP (1.1) contain [7, 9] as special cases.

Funding

The authors are supported financially by the National Natural Science Foundation of China $(11371221,11571296)$.

Competing interests

The authors declare that there is no conflict of interests regarding the publication of this paper.

Authors' contributions

All authors contributed equally and significantly in writing this article. All authors read and approved the final manuscript.

\section{Publisher's Note}

Springer Nature remains neutral with regard to jurisdictional claims in published maps and institutional affiliations.

Received: 7 October 2017 Accepted: 7 February 2018 Published online: 20 February 2018

References

1. Kilbas, A.A., Srivastava, H.M., Trujillo, J.J.: Theory and Applications of Fractional Differential Equations. Elsevier, Amsterdam (2006)

2. Lazarevic, M.P., Spasic, A.M.: Finite-time stability analysis of fractional order time-delay systems: Gronwall's approach. Math. Comput. Model. 49, 475-481 (2009)

3. Delbosco, D.: Fractional calculus and function spaces. J. Fract. Calc. 6, 45-53 (1994)

4. Cabada, A., Wang, G.: Positive solutions of nonlinear fractional differential equations with integral boundary value conditions. J. Math. Anal. Appl. 389, 403-411 (2012)

5. Podlubny, I.: Fractional Differential Equations. Mathematics in Science and Engineering, vol. 198. Academic Press, New York (1999)

6. Bai, Z., Sun, W.: Existence and multiplicity of positive solutions for singular fractional boundary value problems. Appl. Math. Comput. 63, 1369-1381 (2012)

7. Zhang, X., Liu, L., Wu, Y.: The uniqueness of positive solution for a fractional order model of turbulent flow in a porous medium. Appl. Math. Lett. 37, 26-33 (2014)

8. Guo, D., Lakshmikantham, V.: Coupled fixed points of nonlinear operators with applications. Nonlinear Anal. 11(5), 623-632 (1987)

9. Cabada, A., Wang, G.: Nonlinear fractional differential equations with integral boundary value conditions. Appl. Math. Comput. 228, 251-257 (2014)

10. Zhang, X., Liu, L., Wu, Y.: Fixed point theorems for the sum of three classes of mixed monotone operators and applications. Fixed Point Theory Appl. 2016, 46 (2016) 
11. Ahmad, B., Ntouyas, S., Alsaedi, A.: On a coupled system of fractional differential equations with coupled nonlocal and integral boundary conditions. Chaos Solitons Fractals 83, 234-241 (2016)

12. Qarout, D., Ahmad, B., Alsaedi, A.: Existence theorems for semi-linear Caputo fractional differential equations with nonlocal discrete and integral boundary conditions. Fract. Calc. Appl. Anal. 19, 463-479 (2016)

13. Samko, S.G., Kilbas, A.A., Marichev, O.I.: Fractional Integral and Derivative: Theory and Applications. Gordon and Breach, Yverdon (1993)

14. Cao, Z., Jiang, D., Yuan, C.: Existence and uniqueness of solutions for singular integral equation. Positivity 12, 725-732 (2008)

15. Guo, D., Cho, Y., Zhu, J.: Partial Ordering Methods in Nonlinear Problems. Nova Science Publishers, New York (2004)

16. Guo, D., Lakshmikantham, V:: Nonlinear Problems in Abstract Cones. Academic Press, San Diego (1988)

17. Hao, X., Liu, L., Wu, Y.: Iterative solution to singular nth-order nonlocal boundary value problems. Bound. Value Probl. $2015,125(2015)$

18. Henderson, J., Luca, R.: Existence of nonnegative solutions for a fractional integro-differential equation. Results Math. $72,747-763(2017)$

19. Henderson, J., Luca, R.: Existence of positive solutions for a singular fractional boundary value problem. Nonlinear Anal., Model. Control 22, 99-114 (2016)

20. Henderson, J., Luca, R.: Systems of Riemann-Liouville fractional equations with multi-point boundary conditions. Appl. Math. Comput. 309, 303-323 (2017)

21. Guo, L., Liu, L., Wu, Y.: Uniqueness of iterative positive solutions for the singular fractional differential equations with integral boundary conditions. Bound. Value Probl. 2016, 147 (2016)

22. Zhai, C., Hao, M.: Fixed point theorems for mixed monotone operators with perturbation and applications fractional differential equation boundary value problems. Nonlinear Anal. 75, 2542-2551 (2012)

23. Liu, L., Li, H., Liu, C., Wu, Y.: Existence and uniqueness of positive solutions for singular fractional differential systems with coupled integral boundary conditions. J. Nonlinear Sci. Appl. 10, 243-262 (2017)

24. Zhang, X., Liu, L., Wu, Y.: Existence and uniqueness of iterative positive solutions for singular Hammerstein integral equations. J. Nonlinear Sci. Appl. 10, 3364-3380 (2017)

25. Wang, Y., Liu, L.: Uniqueness and existence of positive solutions for the fractional integro-differential equation. Bound. Value Probl. 2017, 12 (2017)

26. Liu, L., Sun, F., Zhang, X., Wu, Y.: Bifurcation analysis for a singular differential system with two parameters via to topological degree theory. Nonlinear Anal., Model. Control 22(1), 31-50 (2017)

27. Wang, Y., Liu, L.: Positive properties of the Green function for two-term fractional differential equations and its application. J. Nonlinear Sci. Appl. 10, 2094-2102 (2017)

28. Liu, L., Zhang, X., Jiang, J., Wu, Y.: The unique solution of a class of sum mixed monotone operator equations and its application to fractional boundary value problems. J. Nonlinear Sci. Appl. 9, 2943-2958 (2016)

29. Liu, L., Zhang, X., Liu, L., Wu, Y.: Iterative positive solutions for singular nonlinear fractional differential equation with integral boundary conditions. Adv. Differ. Equ. 2016, 154 (2016)

30. Liu, L., Hao, X., Wu, Y.: Positive solutions for singular second order differential equations with integral boundary conditions. Math. Comput. Model. 57, 836-847 (2013)

31. Zhang, X., Wang, L., Sun, Q.: Existence of positive solutions for a class of nonlinear fractional differential equations with integral boundary conditions and a parameter. Appl. Math. Comput. 226, 708-718 (2014)

\section{Submit your manuscript to a SpringerOpen ${ }^{\circ}$ journal and benefit from:}

- Convenient online submission

- Rigorous peer review

- Open access: articles freely available online

- High visibility within the field

- Retaining the copyright to your article

Submit your next manuscript at $\gg$ springeropen.com 